Res., Soc. Dev. 2019; 8(10):e498101421

ISSN 2525-3409 | DOI: http://dx.doi.org/10.33448/rsd-v8i10.1421

\title{
Por trás da tela: uso e consequências dos jogos online para multijogadores
}

Behind the screen: use and consequences of online multiplayer games

Detrás de la pantalla: uso y consecuencias de los juegos multijugador en línea

Recebido: 17/07/2019 | Revisado: 06/08/2019 | Aceito: 15/08/2019 | Publicado: 24/08/2019

Paula Argemi Cassel

ORCID: https://orcid.org/0000-0003-2556-4067

Universidade Franciscana, Brasil

E-mail: paula.acassel@gmail.com

Thomaz Girardi Terribile

ORCID: https://orcid.org/0000-0002-0563-565X

Universidade Franciscana, Brasil

E-mail: girarditerribile@gmail.com

Jéssica Costa Machado

ORCID: https://orcid.org/0000-0001-9384-2888

Universidade Federal de Santa Maria, Brasil

E-mail:costamachadojessica@gmail.com

\section{Resumo}

Realizou-se uma revisão assistemática da literatura nacional e internacional, com o objetivo de refletir sobre o uso e as consequências do uso do MMORPG. Buscou-se material teórico nas seguintes bases eletrônicas de dados: Scielo, Google Acadêmico, Pepsic, Scopus, Sciense Direrct, livros, teses e dissertações da CAPES, com os seguintes descritores "videogame", "MMORPG”, “online gaming”, "gaming disorder", “internet”, "behavioral addiction”, sem delimitação de ano de publicação, nas línguas inglês, português e espanhol para isso foram analisados 75 artigos, em duas categorias: Efeitos psicológicos gerais e Gaming disorder. Resultados encontrados sugerem que o MMORPG é um gênero de jogo que produz consequências positivas e negativas, propiciando aquisição de novas amizades, incremento de desempenho acadêmico, bem como incremento de traços de hostilidade, agressividade, dificuldade de resolução de problemas, mediante histórico prévio de funcionamento de personalidade. Ainda, o tempo cronológico despendido no uso de MMORPG aparece como elemento crucial para dependência, apesar dos demais traços. Sugere-se realização de 
pesquisas futuras empíricas envolvendo amostras brasileiras, uma vez que os estudos em nosso país são incipientes.

Palavras-chave: MMORPG; Vício em videogame; Internet; Jogo online.

\begin{abstract}
An asystematic review of the national and international literature was carried out, in order to reflect on the use and consequences of the use of the MMORPG. Theoretical material was used in the following electronic databases: Scielo, Google Scholar, Pepsic, Scopus, Sciense Direrct, books, theses and dissertations of CAPES, with the following descriptors "videogame", "MMORPG", "online gaming" gaming disorder "," internet "," behavioral addiction ", without delimitation of year of publication, in the English, Portuguese and Spanish languages, 75 articles were analyzed in two categories: General psychological effects and Gaming disorder. Results suggest that the MMORPG is a genre of game that produces positive and negative consequences, favoring the acquisition of new friendships, an increase in academic performance, as well as an increase in traits of hostility, aggressiveness, difficulty in solving problems, through a previous history of operation of personality. Also, the chronological time spent in the use of MMORPG appears as a crucial element for dependence, despite the other traits. It is suggested to conduct future empirical research involving Brazilian samples, since the studies in our country are incipient.
\end{abstract}

Keywords: MMORPGs; Gaming disorder; Internet; Online gaming.

\title{
Resumen
}

Se realizó una revisión asistemática de la literatura nacional e internacional, con el objetivo de reflexionar sobre el uso y las consecuencias del uso del MMORPG. Se ha buscado material teórico en las siguientes bases electrónicas de datos: Scielo, Google Académico, Pepsic, Scopus, Sciense Direrct, libros, tesis y disertaciones de CAPES, con los siguientes descriptores "videojuego", "MMORPG", "online gaming", " trastorno de juego "' internet "' adicción conductual ", sin delimitación año de publicación, en los idiomas Inglés, portugués y español para este se analizaron 75 artículos en dos categorías: efectos psicológicos generales y trastorno de juego. Los resultados encontrados sugieren que el MMORPG es un género de juego que produce consecuencias positivas y negativas, propiciando la adquisición de nuevas amistades, incremento de desempeño académico, así como incremento de rasgos de hostilidad, agresividad, dificultad de resolución de problemas, mediante histórico previo de funcionamiento de personalidad. Sin embargo, el tiempo cronológico gastado en el uso de 
Res., Soc. Dev. 2019; 8(10):e498101421

ISSN 2525-3409 | DOI: http://dx.doi.org/10.33448/rsd-v8i10.1421

MMORPG aparece como element crucial para la dependencia, a pesar de los demás rasgos. Se sugiere realizar investigaciones futuras empíricas envolviendo muestras brasileñas, una vez que los estudios en nuestro país son incipientes.

Palabras clave: MMORPGs; Gaming desorden; Internet; Juegos en línea

\section{Introdução}

Com o forte desenvolvimento tecnológico das últimas décadas, principalmente os que concernem à informática, os jogos eletrônicos acabam sendo cada vez mais populares, tornando-se uma das práticas mais importantes dentre as atividades de lazer. (Niemz, Griffiths, \& Bayard, 2005). Tamanho progresso revela um grande alcance na utilização de jogos eletrônicos e estimativas indicam que cada vez mais pessoas façam o uso de produtos tecnológicos nos próximos anos (Cheng, 2014; Kuss \& Griffiths, 2012a).

Nos últimos 25 anos os jogos online se tornaram altamente acessíveis e populares (Kiraly, Nagygyorgy, Griffiths, \& Demetrovics, 2014). O âmbito de jogos eletrônicos passou a ser um dos possíveis entretenimentos, permitindo não só a execução de brincar e jogar como de aprendizagem e habilidades, independente de faixa etária (Palfrey \& Gasser, 2011). O videogame é, então, um jogo eletrônico ou um aplicativo que, através de software e uma tela, criada para fins de entretenimento, também é considerada por professores como ferramenta para a educação, outros consideram ferramenta de distração ou simplesmente como um meio de interação social e até mesmo como um brinquedo (Spada \& Caselli, 2017).

$\mathrm{O}$ videogame pode ser jogado por um controle usando os dedos, em uma ação manipulando os olhos junto com as mãos, ou também com outras partes do corpo. Exemplo disso são os novos jogos para se exercitar, por exemplo, os jogos que simularm exercícios ou danças. Ainda, estes podem ser de duas modalidades: em linha ou rede, é jogado usando a rede em um console fixo ou um computador compartilhando na internet e offline, ele é usado para jogar em um console fixo sem a necessidade de uso da internet (Owen, 2016).

Jogos específicos como o MASSIVE MULTIPLAYER ONLINE (Multijogadores Massivos Online: MMO), surge da conectividade com a internet, ou seja, os primeiros jogos eram desenvolvidos somente para computadores. Com o passar dos anos, começou a serem desenvolvidos também para os consoles (videogames). MMO é uma sigla em inglês que representa uma modalidade, onde vários jogadores se encontram online para competir, socializar e se divertir ao mesmo tempo (Ferrati, 2008). 
Um MMORPG (MASSIVE MULTIPLAYER ONLINE ROLE PLAYNG GAME) pode ser considerado um MMO, porém um MMO nem sempre vai ser um MMORPG. Isso ocorre, pois, o MMO baseia-se em jogos com vários jogadores simultâneos, já o MMORPG possui a mesma característica, porém com um diferencial que é o role playing games. O RPG (Role Playing Games) não possui uma ordem de ações pré-definidas, ou seja, o jogador é livre para explorar todo o mundo virtual, fazer missões, caracterizar seu personagem. Então, fica claro, que o MMORPG é uma fusão dos formatos MMO e RPG.

O modo MMORPG é uma enorme plataforma de comunidade online em que os participantes eles desempenham papel dentro da comunidade online para interagir uns com os outros. Nesse sentido, a estrutura e as normas dos jogos de MMORPG determinam a necessidade de um jogar de forma coletiva. As funções diferem e são organizados conforme cada jogador, diferindo de acordo com a limitação de condução em seus personagens, história, estratégia de jogo e campos de ação. Como eles são jogados? Tudo começa com a escolha de um personagem, chamado avatar, em que cada usuário cria e personifica de acordo com seus gostos. Bem como: armas, roupas, transporte, ou seja, aquilo que pertence à vida real. Ainda, os jogadores podem desfrutar dos benefícios da colaboração entre todos os jogadores (Taylor, 2006).

Todos os MMORPGs tem, por determinação, mundos virtuais imersivos. Os mundos virtuais focam-se particularmente no vínculo com o conteúdo criado e editado pelos utilizadores e pelo chat. Os MMORPGs são focados no progresso de diferentes tipos de personagens e de suas habilidades. O desenvolvimento é utilizado através do combate PvE (player vs. environment, jogador contra o meio ambiente) ou crafting (habilidade de combinar itens do ambiente de jogo para criar armas mais poderosas) e acreditam na socialização e na modificação de avatares e itens que os jogadores podem trocar e vender por moedas virtuais (Rice, 2006; Van Eck, 2010).

$\mathrm{Na}$ personalização dos personagens, cada um conta com diferentes pontos fortes, de fraqueza e, até mesmo, habilidades únicas que podem ser focadas em causar mais danos nos inimigos ou curar a si próprio e sua equipe. A evolução dos personagens são os principais elementos do jogo e se é dada através de um sistema de níveis, onde a cada conquista obtida é recompensada com pontos de experiências que ao final de um determinado número de pontos adquiridos, o avatar aumenta o nível e desbloqueia novos desafios. O progresso vai se tornando gradativamente mais difícil, incentivando os jogadores a jogarem mais para fazer mais missões e assim alcançar novos desafios (Van Eck, 2010). 
ISSN 2525-3409 | DOI: http://dx.doi.org/10.33448/rsd-v8i10.1421

Outra característica deste modo de jogo é que eles são do tipo aberto, onde cada jogador escolhe um caminho para continuar cumprindo atividades ou missões que estão sendo apresentadas na sua jornada, é dito aberto, uma vez que eles têm liberdade para caracterizar seu avatar, além de poder criar outros vários avatares em diferentes contas. Uma vez cumprida essa missão, o jogador recebe algum tipo de incentivo que irá ajudá-lo a fortalecer seu avatar para que continue com a próxima missão (Turkle, 1997).

Nos MMORPGs, as empresas de software estabelecem as regras do jogo, incluindo o quão rápido os personagens podem se mover através do ambiente virtual ou quais armas podem usar. Essas regras, geralmente, permanecem inalteradas pelo jogador. A etiqueta (norma) do jogo é tratada entre os próprios jogadores. Caso não cumpram as regras, há consequências graves para os jogadores, como, outros jogadores atribuírem nomes vulgares aos seus avatares, arriscando ter suas contas banidas ou negociação de itens ou a própria venda da sua conta envolvendo dinheiro real (Ferretti, 2008).

Nesse contexto, os jogos eletrônicos, tanto online ou offline, podem tanger um padrão de mídia, através de seu encanto quase hipnótico, visto como domínio da tecnologia (Kuss \& Griffiths, 2012). Além das utilidades presentes nas novas tecnologias e da perspectiva de imersão em um território virtual através dos jogos eletrônicos, aspecto antes tido como próprio do universo lúdico, tem-se notado regularmente a tendência desfavorável dessa prática, determinada pelo uso patológico de tais artefatos, propondo com isso, uma atenção primordial a essa nova demanda (Spritzer \& Picon, 2012).

No entanto, diversos estudos associam o uso de jogos eletrônicos como promotores de potencial cognitivo e social. Ainda, há identificação dos benefícios de seu uso como válidos em tratamentos médicos e psicológicos (Kim et al., 2006). Porém, de outro ponto de vista, a utilização de jogos eletrônicos é motivo de preocupação para pais e cuidadores, na forma em que se tem estendido o tempo que os jovens estão utilizando os games (Shaw \& Black, 2008). Porém, seu uso, para alguns indivíduos, pode acarretar dano à saúde, tanto mental quanto física, no âmbito acadêmico bem como nos relacionamentos afetivos (Tao et al., 2010). O tempo excessivo gasto, então, em mídia virtual, definitivamente desloca o individuo de outras atividades de vida, o que resulta em consequências negativas. De qualquer forma, frisa-se que ademais do tempo gasto, as variáveis psicológicas e cognitivas desempenham importante papel na compreensão dos indivíduos que jogam online (Liu \& Peng, 2009).

$\mathrm{O}$ crescimento acentuado do uso de jogos eletrônicos tem ampliado a preocupação acerca das problemáticas emergentes que envolvem o jogo online (Demetrovics \& Griffiths, 2012; Kuss \& Griffiths, 2012b). Nesse ensejo, os problemas associados aos jogos eletrônicos 
propendem a continuar ao longo do tempo para a maioria das pessoas afetadas. Vale ressaltar que há altíssima predominância de comorbidades psiquiátricas associadas a essa condição (Tao et al., 2010). A pesquisa sobre dependência de jogos eletrônicos teve aumento de interesse nos últimos anos, tendo foco em como a fenomenologia desse tema, etiologia e a classificação desse comportamento de dependência. Assim, a literatura disponível sobre o tema apresenta aspectos que são relevantes para o âmbito da clinica, tais como: tratamento de jogos eletrônicos, perfil de comorbidade e associação dos jogos violentos com o comportamento agressivo (McClellan \& Dorn, 2008).

O Manual Diagnóstico e Estatístico dos Transtornos Mentais (DSM-5) adiciona a categoria "Internet Gaming Disorder" (GD) como doença, suscitando maior interesse e foco de atenção para tal temática (Santaella, 2009). A inserção no DSM-5 demarca o uso abusivo dos indivíduos, em diferentes contextos e idades, no envolvimento com os jogos eletrônicos, o que tende a acarretar em prejuízos significativos na vida do sujeito, tornando-se, assim objeto de pesquisa de importância ímpar para a psicologia, a fim de que se possa compreender os processos subjetivos envolvidos no uso de jogos eletrônicos.

Salienta-se que o GD é uma das categorias expressas em um espectro de adição em internet; os demais se referem a outras atividades como compras, jogos de azar ou rede sociais. Então, GD representa uma parte do constructo de adição em internet, sendo o GD o fenômeno com maiores investimentos de investigação cientifica no que se refere ao espectro de adição em internet (Kuss \& Griffiths, 2012b).

Dito isso, a popularidade do MMORPGs aponta a importância de se investigar como estes impactam na vida dos jogadores (Zhog \& Yao, 2012). A maioria dos estudos existentes sobre MMORPGs focam-se em investigar as possibilidades da adição causada por eles (Lee, Yu, \& Lin, 2007). Apesar disso, algumas investigações têm prestado atenção para os aspectos sociais do MMORPGs (Ducheneaut, Yee, Nickell, \& Moore, 2006). Assim, este artigo tem como objetivo analisar por meio de uma revisão assistemática da literatura científica nacional e internacional o uso dos jogos com o gênero MMORPG e suas consequências psicológicas.

\section{Metodologia}

A pesquisa realizada foi de revisão assistemática da literatura, e elaborada, a partir de materiais já publicados que envolvem o tema proposto (Prodanov e Freitas, 2013). A busca pelos materiais ocorreu nas seguintes bases eletrônicas de dados: Scielo, Google Acadêmico, Pepsic, Scopus, Sciense Direrct, livros, teses e dissertações da CAPES, com os seguintes 
descritores "videogame", "MMORPG", "online gaming”, "gaming disorder", "internet", "behavioral addiction", sem delimitação de ano de publicação, nas línguas inglês, português e espanhol. Ainda, houve busca ativa nas referências dos artigos encontrados. A coleta de dados foi realizada no período de março a junho de 2019.

Para análise dos dados coletados, foi utilizada a metodologia da Análise de Conteúdo, que é um conjunto de técnicas de investigação que, por meio de uma descrição sistemática, objetiva e quantitativa do conteúdo oriundo das comunicações, objetivando interpretar tais mensagens (Bardin, 2009). A análise, conforme indicação do método, foi dividida em três momentos; o primeiro de pré-análise, no qual houve a realização de leitura flutuante de documentos acerca da temática, após a escolha destes documentos. Na segunda etapa houve a exploração do material, com sua codificação, e por fim, o tratamento dos resultados, a inferência e a intepretação.

Foram encontrados, para a execução desse artigo, através dos descritores citados acima, entre todas as plataformas de dados pesquisados, artigos correspondentes. Assim, realizou-se uma seleção dos documentos encontrados, a partir da leitura de seus respectivos resumos. Após descartar os que não possuíam temáticas relacionadas com o objetivo deste artigo, restaram 17 escritos. Após, leitura completa dos artigos foram retirados quatro artigos, restando 13 artigos. Por último, foi feita a leitura completa dos documentos para a elaboração deste trabalho. Foram, então, incluídos artigos encontrados nas referências destes iniciais, totalizando para análise documentos. Após leitura de todos documentos, foram criadas duas categorias, a partir de dois juízes, para apresentação dos resultados: efeitos psicológicos gerais e gaming disorder. A primeira categoria compõe-se por 29 artigos; e 46 fazem parte da segunda. Dois artigos foram incluídos em ambas as categorias, uma vez que apresentam resultados de consequências gerais (Smyth, 2007; Spada \& Caselli, 2017).

\section{Resultados e discussões}

\section{Efeitos psicológicos gerais}

Os jogos online possuem características específicas, tais como, interação com outras pessoas, fazer uso de avatar, possibilitando que haja conexões e vínculos que extrapolam a máquina (Turkle, 1997). O mundo virtual, proporcionado pelos jogos do gênero MMORPG, se encontra em continua evolução e é moldado por todos os jogadores, ou seja, implica na compreensão que o jogo é para além do que um jogo simplório, pois oferece a oportunidade de exercer interação em um mundo fantasioso, no qual o indivíduo pode se afastar do mundo 
real, explorar espaços novos, batalhar com inimigos, ser reconhecido como herói, se conectar com pessoas a nível mundial, aprender um novo idioma, fazer negócios, aprender e resolver problemas, tudo isso em tempo real, sozinho ou em equipe (Kelly, 2004).

Ainda, os jogos online desse gênero apostam, de forma intensa, no componente social, e na jogabilidade, algo fundamental para manter os jogadores interessados por semanas, meses ou anos para que sempre haja diversidade e deixem de abandonar o mundo online (Rice, 2006; Van Eck, 2010). Desse modo, é possível inferir que o jogo MMORPG e seus jogadores estão em constante conexão, impossibilitando que haja um fim estipulado ou uma ideia de quando ele possa terminar, ou seja, há contínuo funcionamento mesmo quando os jogadores não se encontram no espaço virtual: como no trabalho, dormindo ou realizando outra atividade (Kelly, 2004).

Os jogos MMORPG tem sido associados com maior capacidade de aprendizado, desenvolvimento de habilidades motoras, afetivas e cognitivas juntamente com a desenvoltura para uma melhor socialização. Muitos benefícios de seu uso têm sido validados também em tratamentos médicos e psicológicos (Kim et al., 2006). Com o crescente uso da tecnologia, diferentes estudos estão sendo realizados, em específico um deles foi realizado com jovens estudantes, residentes de casa estudantis, teve por objetivo verificar o aprendizado que este público pode possuir com os jogos do gênero MMORPGS. Os resultados indicaram que há presença de aspectos psicológicos de interação social, outras formas de aprendizado e concentração (Sarsar, 2008).

Diferentes pesquisas têm se concentrado em investigar dinâmicas sociais estabelecidas entre os jogadores de MMORPG. Em maioria, os resultados sugerem que os MMORPGs envolvem formas complexas de interação e colaboração entres si, as quais se traduzem no aprimoramento de habilidades sociais e atividades na vida real (Axelsson \& Regan, 2002; Duchenaut \& Moore, 2005; Heckel, 2003; Oliver, 2002; Whang, 2003; Williams et al., 2006; Wing, 2007). É preciso considerar, que outras investigações, de forma inversa, encontraram que interações virtuais fechadas (somente entre estes indivíduos), provocadas pelo MMORPG, comumente, são trocadas e escolhidas, deixando-se de lado as relações presenciais; apesar disto, há o desenvolvimento, na vida real, a partir do aprendizado no jogo, habilidades relacionadas ao empreendedorismo, finanças e economia (Dobson, 2006; Lehdonvirta, 2007).

Do mesmo modo, foi demonstrado, por um estudo experimental, comparando grupos de jogadores online de diferentes gêneros, que jogadores MMORPGs possuem maior socialização em vida real e bom desempenho acadêmico. Ainda, esse grupo de jogadores 
Res., Soc. Dev. 2019; 8(10):e498101421

ISSN 2525-3409 | DOI: http://dx.doi.org/10.33448/rsd-v8i10.1421

reportam maior engajamento no jogo, maior interesse em continuar jogando, e maior aquisição de novas amizades (Smyth, 2007).

Nos aspectos motivacionais e emocionais, alguns autores encontraram que aspectos sociais e culturais do MMORPG são pilares de incentivo para jogar, provocando emoções agradáveis e forte união entre os jogadores (Chen, Duh, Puah, \& Lam, 2006; Cole \& Grifiths, 2007). Ainda, a estrutura de base e natureza sociocultural o MMORPG se insere está associado a um maior nível de inserção na era digital com níveis mais altos de desempenho lógico-numérico e incremento das habilidades escolares (Souza, Lima e Silva, \& Roazzi, 2015).

Os MMORPGs podem oferecer aos jogadores adultos diversas oportunidades para manutenção e o desenvolvimento de relacionamentos com suporte e significado afetivo. Um dos fatores nesse sentido é o estudo do capital social (Zhang \& Kaufman, 2016); este possui diferentes conceitos, mas todos englobam um fator primordial: networking, ou seja, contato, rede de relações (Lee \& Lee, 2010; Trepte, Reinecke, \& Juechems, 2012). Em uma pesquisa foi encontrado que há envolvimento de relações, com conceito de capital social, com impactos profundos nos adultos associado aos efeitos positivos ao jogo, pois estes indivíduos são mais dependentes no contexto de relacionamento do jogo (Zang \& Kaufman, 2015).

Outro estudo compara o capital social em jogadores online e offline. Este estudo contribui para o conhecimento do capital social porque testa os efeitos das novas mídias sobre o capital social online e offline na cultura chinesa. Além disso, este estudo fornece evidências empíricas para os efeitos positivos dos jogos on-line e destaca a experiência social nos jogos MMORPG e como isso influencia as redes sociais dos jogadores e a participação coletiva (Zhong, 2011). Diante do exposto, pensar que as questões afetivas e sociais estão intimamente ligadas ao contexto dos jogos de forma positiva, implica em novos patamares de utilização dos mesmos, como em contextos educacionais, onde a socialização é parte crucial do processo de ensino-aprendizagem. Além disso, como mencionado anteriormente por Kim et al. (2006), os jogos tem uma capacidade de melhora na qualidade de aprendizagem, de habilidades motoras, o que corrobora a ideia de sua utilização no meio educacional.

Por outro lado, na investigação de Zhong (2011), na busca das consequências do uso de jogos online, dois modelos de equações estruturais foram desenvolvidos para testar se o jogo coletivo influencia o capital social offline através da mediação do capital social online. Os resultados não demonstraram a existência de efeitos de mediação. Especificamente, o jogo coletivo influencia positivamente o capital social dos vínculos on-line dos jogadores, o capital social online e o engajamento social online. $\mathrm{O}$ efeito do jogo coletivo no relacionamento 
offline e na união do capital social não é significativo; o efeito do capital social vinculado / vinculativo online no capital social vinculado e/ou vinculativo offline também não é significativo. O estudo encontra um impacto significativamente positivo do jogo coletivo no engajamento cívico offline. O efeito do engajamento social online no engajamento social offline não é significativo. Em contraste com o jogo coletivo, o tempo de jogo influencia negativamente o capital social online e offline.

Igualmente, sabe-se que os jogadores de MMORPGs não realizam esta atividade de forma solitária e, justo por isso, possuem incremento nas experiências sociais (Caplan, Williams, \& Yee, 2009; Ducheneaut \& Moore, 2004). Em pesquisa sobre o desenvolvimento e as propriedades psicométricas de uma escala sobre metacognição sobre jogos online, desenvolvida, a partir de dois amostras, com 225 e 348 participantes, de jogadores online, examinou a capacidade da escola em predizer as horas de jogo online com adição a internet. Como um dos resultados foi evidenciado que a consistência interna, validade preditiva e divergente foram aceitáveis. Todos os fatores de metacognição da escala se correlacionaram positivamente com as horas semanais de jogo online e o vício em Internet (Spada \& Caselli, 2017), o que sugere que fator protetivo seria redução de números de horas de jogo.

Os achados indicam que há tanto consequências positivas quanto negativas em relação ao uso de jogos online. As consequências positivas aparecem em maior quantidade e com diversidade. Se por um lado esses jogos estimulam o aprendizado e processos de ensinoaprendizagem, colaborando de maneira significativa para a socialização, por outro lado, seu uso inadequado pode afetar de maneira inversa, alguns dos ganhos já mencionados, como ansiedade social e isolamento. Torna-se importante pensar em estratégias efetivas que possam conduzir os usuários de jogos online ao uso saudável dessa ferramenta tecnológica, assim como, á estimulá-los a apreciação de jogos que além socialização efetiva, possam colaborar para utilização de outros processos cognitivos e afetivos que estejam carentes nos jogadores.

\section{Gaming disorder (GD)}

Pesquisas recentes têm demonstrado que jogar online é uma das maiores razões associadas ao uso problemático da internet (Ducheneaut \& Moore 2004; Meerkerk, Van Den Eijnden, \& Garretsen, 2006). Do mesmo modo, outras pesquisas sugerem que o uso excessivo de internet pode levar ao desenvolvimento de comportamento aditivo (Young, 2009; Muller \& Wolfling, 2011). Existem evidências clinicas de que a adição em internet está associada à adição de outras substancias, modificações no humor, com sintomatologia de crise de 
abstinência, e dificuldades no funcionamento da tolerância, na resolução de conflitos, e manifestações de comportamentos relapsos (Griffiths, 2005).

$\mathrm{O}$ aproveitamento de jogos eletrônicos, associado à psicopatologia, pode ser visto como uma prática desadaptativa. Um dos indícios de uso excessivo na utilização de tais tecnologias ocorre quando o comportamento se torna aditivo, ou seja, prejudica o sujeito de forma que ele se torne incapaz de conter a periodicidade perante um comportamento que antes era visto inofensivo (Peukert, Sieslack, Barth, \& Batra, 2010).

O uso excessivo de internet pode ser associado a uma variedade de consequências psicossociais negativas. Estas incluem desordens mentais como somatização, traços obsessivos compulsivos, transtornos de ansiedade, depressão, dissociação (Adalier \& Balkan, 2012; Bernardi \& Pallanti, 2009), bem como traços de personalidade e patologias como introversão e psicoticismo (Xiuqin et al., 2010). Há ainda, indicadores apontados na literatura de que o uso incontrolável de jogos online acarreta em diferentes possibilidades de sintomatologias comportamentais e cognitivas similares àquelas geradas pelos transtornos psiquiátricos de uso de substâncias (Ko et al., 2014). Igualmente, é preciso compreender de forma mais clara, o que ainda é uma lacuna nas pesquisas encontradas, como os mecanismos de adição pelos jogos online ocorrem, a partir das comorbidades psiquiátricas apresentadas por estes indivíduos (Buckley \& Brown, 2006; Kessler, 2004).

GD aparece como uma grande influência nos aspectos físico, mental e sociais a saúde devido ao excesso da adição em jogos da internet (Sakuma et al., 2017). A adição à internet possui maior prevalência entre adultos jovens (Bakken, Wenzel, Gotestam, Johansson, \& Oren, 2009). Os indivíduos com dependência têm um alto risco de comorbidades (Hogue, Henderson, \& Schmidt, 2016; Marquez-Arrico, Lopez-Vera, Prat, \& Adan, 2016). Comorbidades como transtornos do desenvolvimento e de ansiedade prejudicam o uso de estratégias de enfrentamento dos indivíduos, o que provoca menor autoeficácia em transtornos aditivos (Hermsen et al., 2016). Além disso, as comorbidades estão relacionadas à gravidade do GD (Baggio et al., 2016; Han et al., 2015; Wei, Chen, Huang, \& Bai, 2012).

O diagnóstico de transtorno de GD é correlacionado com transtorno de déficit de atenção e hiperatividade (Batthyany, Muller, Benker, \& Wolfling, 2009; Carli, Durkee, Wassernan, et al., 2013; Ko, Yen, Chen, Chen, \& Yen, 2008), sendo ambos associados a características de impulsividade e hostilidade (Yen et al., 2017). Pormenor, uma pesquisa que investiga tais itens sugere que adultos jovens que possuem GD tem associação diagnóstica de TDAH, como referido. No entanto, os resultados apresentados são salientes ao indicar que os adultos jovens que possuem ambos os diagnósticos possuem maiores níveis de impulsividade 
Res., Soc. Dev. 2019; 8(10):e498101421

ISSN 2525-3409 | DOI: http://dx.doi.org/10.33448/rsd-v8i10.1421

e hostilidade (Yen et al., 2017). Ou seja, estas características podem ser fortalecedoras para a associação de GD com TDAH, o que faz com que se necessite dar maior atenção a crianças e adolescentes que apresentem tais características de maneira intensa, e para que possa-se então trabalhar com a prevenção, identificação e desenvolvimento de tais transtornos.

Outros estudos revelam que assim como no transtorno de uso de substâncias, a impulsividade possui papel primordial para o GD (Ding, Sun, Sun, et al., 2014; Ko, Hsieh, Wang, et al., 2015). Seguindo na mesma linha, referente à hostilidade, outras pesquisas sugerem que essa se caracteriza por ser um sintoma psiquiátrico que se reflete em características de isolamento ligadas ao afeto, à cognição, e ao comportamento, sendo essa associada ao GD (Choo et al., 2010; Lin et al., 2008; Yen, Ko, Yen, Wu, \& Yang, 2007).

Na mesma direção, corroborando os achados acima, um estudo de dois anos realizado com pessoas de 16 a 18 anos, na Grécia, em sala de aula comprova que os jogos de MMORPG geraram mais hostilidade em adolescentes que já possuíam vício em internet. Entretanto, uma porcentagem maior de jogadores aparentou ter uma proteção em relação ao potencial de vício em internet (Stavropoulos et al., 2015), o que enfatiza a importância dos fatores contextuais com diferença nos sintomas do vício na adolescência, pois a ausência de limites para o uso pode deixar as pessoas mais hostis de modo que sua expressão seja de forma agressiva offline e não seja aceita pela sociedade (Ko et al., 2009).

Ainda nesse contexto, considera-se como categoria possível, então, de diagnóstico o uso problemático de jogos online, que se caracteriza pela frequência do jogo ser maior do que a planejada, causando negligencia de aspectos do cotidiano; pelo impacto negativo nas relações e interações sociais; e, na redução sintomática de outras questões (Peters \& Malesky, 2008). De 3 a 10\% é a prevalência de uso problemático de jogos online (Kuss, Griffiths, Krila, \& Billlieux, 2014), com presença altos níveis de comorbidade de transtorno de déficit de atenção (Batthyany, Muller, Benker, \& Wolfling, 2009), bem como depressão e ansiedade social (Gentile et al., 2011; Peng \& Liu, 2010).

A etiologia e o progresso de adição aos jogos online, como já referido, encontram-se associados aos fatores críticos de interação social e impulsividade (Liu \& Peng, 2009). A partir dessa constatação, um artigo investigou as repercussões de tais fatores, comparando três tipos de gêneros de jogos online, MMORPG, RTS (real-time strategy) e FPS (first-person shooter) e evidenciaram que o tipo de gênero não está associado com impulsividade, mas o gênero está associado aos fatores de sociabilidade, indicados por ansiedade social, autoestima, e envolvimento familiar. em comparação as demais categorias analisadas; ainda, os jogadores 
ISSN 2525-3409 | DOI: http://dx.doi.org/10.33448/rsd-v8i10.1421

de MMORPG possuíam o maior nível de ansiedade social em comparação com os demais gêneros (Park et al., 2016).

Em um estudo sobre videogame conduzido por $\mathrm{Ng}$ e Wiemer-Hastings (2005) encontrou que jogadores de MMORPG possuem maiores problemas de uso do que jogadores de videogame offline, incluindo jogar por mais de oito horas seguidas, perda de sono. Também foi demonstrado na pesquisa citada que os jogadores de MMORPG gastam muito tempo jogando, o que provoca um menor tempo com amigos offline, bem como desvalorizamento de relacionamento social offline.

Em um estudo experimental sobre as consequências do jogar videogame foi identificado que jogadores de MMORPG diferem de forma significativa de outros jogadores de outro gênero em relação a horas jogadas, apresentam pior saúde, pior qualidade de sono (Smyth, 2007). Ainda, foram evidenciados por uma pesquisa (Spada \& Caselli, 2017) através de análises de regressão que metacognições negativas de incontrolabilidade dos jogos online e os níveis de dependência da internet foram os únicos preditores significativos das horas semanais de jogo, ao passo que metacognições positivas sobre jogos online e metacognições negativas sobre a incontrolabilidade dos jogos online foram os únicos preditores significativos de vício em internet.

Sabe-se que os traços de personalidade podem desempenhar um papel na adição, uma vez que diferentes pessoas parecem ter personalidade que os leva à adição (Griffiths, 2009). As relações entre traços de personalidade, especificamente de busca de emoções, autocontrole, agressão, neuroticismo, estado de ansiedadec e traços de ansiedade em relação a jogadores online foram evidenciadas por meio de uma regressão linear múltipla na pesquisa de Mehroof e Griffiths (2010), na qual exibiram associações significativas com vício em jogos. O estudo sugere que certos traços de personalidade podem ser importantes na aquisição, desenvolvimento e manutenção do vício. Nesse sentido, o auto controle parece influenciar o indivíduo a se manter no jogo online (Mehroof \& Griffiths, 2010).

$\mathrm{O}$ traço de neuroticisimo indica que uma pessoa possui maior predisposição à ansiedade geral e tendências a preocupação. Nesse sentido, estudos observam e sugerem que jogos online possuem impactos negativos no bem-estar geral e que indivíduos com traços marcantes de neuroticismo são os mais propensos, em comparação a outros indivíduos, com outros traços de funcionamento de personalidade, a serem adictos a jogos online (Biao-Bin et al., 2006; Huh \& Bowman, 2009).

Ainda em relação a personalidade, indivíduos que se colocam em risco e experenciam diferentes atividades consideradas normais, aqueles que se propõem a se engajarem em 
comportamentos novos, complexos e intensos, de certo modo patológicos em razão de suas características, podem possuir alto risco de desenvolver dependência em jogos online (Wan \& Chiou, 2006). Igualmente, traços de agressividade também parecem possuir impacto no uso dos jogos online; pesquisas demonstram que adolescentes meninos têm maior atração por jogos violentos em relação aos não-violentos, bem como demonstração do link entre jogar excessivamente e ter apreço por jogos violentos (Ko et al., 2009).

Se por um lado o vício em internet prejudica a vida e as relações das pessoas, por outro lado o gênero de jogo online MMORPG funcionam como um catalisador para o contato social e pode complementar as relações face-a-face, potencializando efeitos benéficos do jogo (Jones et al., 2014). Portanto, para uma pessoa que joga com seus amigos da vida real, a socialização seria maior em comparação com um jogador isolado, possivelmente diminuindo o risco de vício em internet (Stavropoulos et al., 2015). A maioria dos estudos aqui evidenciados sobre o uso do excessivo da internet com jogos apresentaram resquícios negativos, porém o gênero MMORPG pode colaborar de maneira significativa para um menor prejuízo social. O que nos leva a pensar que os jogos, mesmo que usados de forma inadequada, possam ainda, exercer papel colaborativo na vida das pessoas.

\section{Considerações finais}

O presente artigo objetivou refletir sobre o uso de jogos online, especialmente do gênero MMORPG, e suas consequências. A partir das reflexões realizadas ao longo do trabalho pode-se perceber que o recente desenvolvimento de jogos online, socialmente integrados do gênero MMORPGs tem criado uma nova forma de experiência para os jogadores. Os MMORPGs representam uma experiência de jogo diferente com diversas consequências, não inseridas em outros tipos de gêneros de jogos e parece possuir tanto riscos como benefícios para aqueles que fazem seu uso. Um aspecto importante evidenciado no presente estudo é que, o uso desse gênero de jogo online contribui para a interação e interdependência social, que podem impactar substantivamente o desenvolvimento de habilidades sociais e emocionais entre os indivíduos que optam por experienciar o jogo. Além disso, foi evidenciado que, em conjunto com a desenvoltura para uma melhor socialização, esse gênero de jogo pode trazer benefícios como ampliação da capacidade de aprendizado, desenvolvimento de habilidades motoras, afetivas e cognitivas. Esses resultados apresentam importante contribuição, visto que estas habilidades estimuladas pelo jogo são deficitárias na população geral e também estão presentes em transtornos como de ansiedade, o que nos faz 
ISSN 2525-3409 | DOI: http://dx.doi.org/10.33448/rsd-v8i10.1421

pensar que os jogos podem contribuir de maneira significativa para fortalecer as habilidades já mencionadas. Esses achados fortalecem-se pelos apontamentos como de Kim et al. (2006), que trazem contribuições sobre validação desses jogos em tratamentos médicos e psicológicos. Pensa-se que, além de trabalhar questões psicológicas, como o treino de habilidades sociais em ambiente virtual, esses jogos, por possibilitarem uma melhora na capacidade de aprendizado, podem também colaborar para atendimento educacional especializado, como no caso dos transtornos de aprendizagem.

Se por um lado os MMORPGs criam um ambiente virtual atraente, imersivo e socialmente rico, por outro, à medida que mais pessoas se tornam imersas em sociedades virtuais, é importante que continuemos examinando os custos e benefícios da participação nesses mundos. Outro achado relevante desse estudo é que horas semanais de jogo online relacionou-se ao vício em Internet (Spada \& Caselli, 2017) e que as características específicas dos indivíduos, como ansiedade social, parecem estar fortemente associadas a problemas relacionados a jogos, semelhante a questões aditivas no grupo MMORPG. Desta maneira, verificasse que existem consequências do uso de jogos online devido a características de personalidade, especialmente quando o número de horas que o jogador fica em frente ao vídeo game é excessivo. Uma estratégia eficaz nesses casos, seria o atendimento psicológico para verificação de gatilhos no tempo e consequente uso exacerbado dos jogos, além de estratégias de auto monitoramento. Em conjunto com o atendimento psicológico, torna-se relevante o monitoramento do número de horas de jogo, realizado por pais ou cuidadores de crianças e adolescentes.

Ainda, é crucial considerar que os resultados apresentados neste trabalho se circunscrevem a realidades não brasileiras, uma vez que há uma carência intensa de estudos em nosso país acerca da temática. Para futuros trabalhos devem-se considerar a realização de pesquisa empírica, a fim de identificar, em amostra brasileira, os benefícios de jogar MMORPGs (ou até mesmo o desenvolvimento de jogos terapêuticos ou de construção de habilidades semelhantes aos MMORPG), juntamente com os riscos e custos potenciais.

\section{Referências}

Adalier, A. \& Balkan, E. (2012). The relationship between Internet addiction and psychological symptoms. International Journal of Global Education. 1(2): 42-49. 
Axelsson, Ann-S. \& Regan, T. (2002). How Belonging to an Online Group Affects Social Behavior - a Case Study of Asheron's Call. Redmond, Washington: Microsoft Research.

Bakken, I. J., Wenzel, H. G., Gotestam, K. G., Johansson, \& Oren (2009). Internet addiction among Norwegian adults: A stratified probability sample study. Scandinavian Journal of Psychology. 50(1): 121-127.

Baggio, S., Dupuis, M., Studer, J., Spilka, S., Daeppen, J.-B., Simon, O. \& Gmel, G. (2016). Reframing video gaming and Internet use addiction: Empirical cross-national comparison of heavy use over time and addiction scales among young users. Addiction, 111, 513-522. doi: 10.1111/add.13192.

Batthyany, D., Muller, K.W., Benker, F., \& Wolfling, K. (2009). Computer game playing: Clinical characteristics of dependence and abuse among adolescents.

Buckley, P. F., \& Brown, E. S. (2006). Prevalence and consequences of dual diagnosis. The Journal of Clinical Psychiatry, 67(7), e01

Caplan, S.E., Williams, D., \& Yee, N. (2009) Problematic Internet use and psychosocial wellbeing among MMO players. Computers in Human Behavior. 25(1):1312-19. doi:10.1016/j.chb.2009.06.006

Chen, V. H., Duh, H. B., Puah, P. S. K., \& Lam, D. Z. (2006). Enjoyment or engagement? Role of social interaction in playing massively multiplayer online role-playing games (MMORPGS). In Entertainment computing - ICEC 2006 (pp. 262-267). Berlin, Germany: Springer. doi:10.1007/11872320_31

Cheng, C. \& Li, Ay-L. (2014). Internet addiction prevalence and quality of (real) life: a metaanalysis of 31 nations across seven world regions. Cyberpsychology, Behavior, and Social Networking. Dec, 17(12). 
Choo, H., Gentile, D. A., Sim, T., Li, D., Khoo, A., \& Liau, A. K. (2010). Pathological videogaming among Singaporean youth. Annals of the Academy of Medicine, Singapore, 39(11): 822-829.

Cole, H., \& Griffiths, M. D. (2007). Social interactions in massively multiplayer online roleplaying gamers. CyberPsychology \& Behavior, 10(1):575-583. doi:10.1089/cpb.2007.9988

Demetrovics, Z., \& Griffiths, M. D. (2012). Behavioral addictions: Past, present and future. Journal of Behavioral Addictions, 1(1): 1-2.

Ding, W. N., Sun, J. H., Sun, Y. W., et al. (2014). Trait impulsivity and impaired prefrontal impulse inhibition function in adolescents with Internet gaming addiction revealed by a go/no-go fMRI study. Behavioral and Brain Functions: BBF, 10, 20. http://dx.doi. org/10.1186/1744-9081-10-20.

Dodson, E. (2006). Das virtual capital: An economic perspective on playing online games, fantastic scarcity, and real-market trades. Oregon: Bacharelado em Economia de Recursos, Agrária e do Ambiente, Oregon State University, Corvallis.

Ducheneaut, N. \& Moore, R.J. (2004). Gaining more than experience points: Learning social behavior in multiplayer computer games. In: Conference proceedings on human factors in computing systems (CHI2004): Extended Abstracts, April 24-29, 2004, Vienna, Austria.

Ducheneaut, N. \& Moore, R.J. (2005). The social side of gaming: a study of interaction patterns in a massively multiplayer online game. In: Proceedings of CSCW 2004, ACM, New York, p. 360-369.

Ducheneaut, N., Yee, N., Nickell, E., \& Moore, R. J. (2006). "Alone Together?" Exploring the social dynamics of massively multiplayer online games. In: Proceedings of CHI 2006, ACM, New York, p.6.

Durkee, T. et al. (2012). Prevalence of pathological internet use among adolescents in Europe: demographic and social factors. Addiction. 26(1). 
Res., Soc. Dev. 2019; 8(10):e498101421

ISSN 2525-3409 | DOI: http://dx.doi.org/10.33448/rsd-v8i10.1421

Ferretti, S. (2008). Cheating detection through game time modeling: A better way to avoid time cheats in P2P MOGs? Multimedia Tools and Applications. 37(1):339-63.

Gentile, D.A., Choo, H., Liau, A., Sim, T., Li, D., Fung, D., \& Khoo, A. (2011). Pathological Video Game Use Among Youths: A Two-Year Longitudinal Study. Pediatrics, 127(2): 319329. doi: $10.1542 /$ peds.2010-1353

Gentile, D.A., Choo, H., Liau, A., Sim, T., Li, D.D., Fung, D., et al. (2011). Pathological video game use among youths: A two-year longitudinal study. Pediatrics, 127(1): 319-329.

Griffiths, M. D. (2005). A components model of addiction within a biopsychosocial framework. Journal of Substance Use, 10(4): 191-197.

Heckel, H. L. (2003). Online social interaction: The case of Everquest. (Tese para graduação da Faculty of George Mason University para Grau de Mestre em Arts Sociology). (Autores, vocês fecham o parágrafo no final, mas onde é ele se inicia????? Verifiquem. Acertem. Agradecemos).

Hogue, A., Henderson, C. E., \& Schmidt, A. T. (2016). Multidimensional predictors of treatment outcome in usual care for adolescent conduct problems and substance use. Administration and Policy in Mental Health and Mental Health Services Research. http://dx.doi.org/10.1007/s10488-016-0724-7

Kelly, R. V. (2004). Massively Multiplayer Online Role Playing Games, Ed. McFarland \& Company Inc. Publishers.

Kessler, R. C. (2004). The epidemiology of dual diagnosis. Biological Psychiatry, 56(10): 730-737. http://dx.doi.org/10.1016/j.biopsych.2004.06.034.

Kessler, R. C. (2004). The epidemiology of dual diagnosis. Biological Psychiatry, 56(10):730-737. http://dx.doi.org/10.1016/j.biopsych.2004.06.034. 
Kim, K., Ryu, E., Chon, M. Y., Yeun, E. J., Choi, S. Y., Seo, J. S. \& Nam, B. W. (2006). Internet addiction in Korean adolescents and its relation to depression and suicidal ideation: A questionnaire survey. International Journal of Nursing Studies, 43(2):185-192.

Ko, C. H., Hsieh, T. J., Wang, P. W., et al. (2015). Altered gray matter density and disrupted functional connectivity of the amygdala in adults with Internet gaming disorder. Progress in Neuro-Psychopharmacology \& Biological Psychiatry, 57(1):185-192. http://dx.doi.org/10.1016/j.pnpbp.2014.11.003.

Király, O., Nagygyörgy, K., Griffiths, M. D., \& Demetrovics, Z. (2014). Problematic online gaming. In K. Rosenberg \& L. Feder (Eds.), Behavioral addictions: Criteria, evidence and treatment (pp. 61-95). New York, NY: Elsevier.

Ko, C. H., Yen, J. Y., Chen, C. S., Chen, C. C., \& Yen, C. F. (2008). Psychiatric comorbidity of internet addiction in college students: an interview study. CNS Spectr, 13(1):147-153.

Ko, C. H., Yen, J. Y., Chen, C. S., Yeh, Y. C., \& Yen, C. F. (2009). Predictive values of psychiatric symptoms for Internet addiction in adolescents: A 2-year prospective study. Archives of Pediatric and Adolescent Medical, 163(1): 937-943. doi:10.1001/archpediatrics.2009.159

Kuss, D. J. \& Griffiths, M. D. (2012a). Online gaming addiction in children and adolescents: a review of empirical research. Journal of Behavioral Addictions. 29(1):3-29.

Kuss, D. J., \& Griffiths, M. D. (2012b). Internet gaming addiction: A systematic review of empirical research. International Journal of Mental Health and Addiction, 10(2):278-296.

Kuss, D. J., Griffiths, M. D., Karila, L., \& Billieux, J. (2014). Internet addiction: A systematic review of epidemiological research for the last decade. Current Pharmaceutical Design, 20(1):4026-4052.

Ko, C.-H., Yen, J.-Y., Chen, S.-H., Wang, P.-W., Chen, C.-S., \&Yen, C.-F. (2014). 
Res., Soc. Dev. 2019; 8(10):e498101421

ISSN 2525-3409 | DOI: http://dx.doi.org/10.33448/rsd-v8i10.1421

Evaluation of the diagnostic criteria of Internet gaming disorder in the DSM-5 among young adults in Taiwan. Journal of Psychiatric Research, 53(1):103-110. doi:https://doi.org/10.1016/j.jpsychires.2014.02.008

Ko, C. H., Hsieh, T. J., Wang, P. W., et al. (2015). Altered gray matter density and disrupted functional connectivity of the amygdala in adults with Internet gaming disorder. Progress in Neuro-Psychopharmacology \& Biological Psychiatry, 57(1):185-192. http://dx.doi.org/10.1016/j.pnpbp.2014.11.003.

Lee, I., Yu, C-Y., Lin, H (2007). Leaving a Never-Ending Game: Quitting MMORPGs and Online Gaming Addiction. DIAGRA, Situated Play.

Lehdonvirta, V. (2007). Virtual economics: Applying economics to the study of game worlds. Available em: www.strategicleader.us/ ExperientalLearningPapers/Papers/paper236_lehdonvirta.pdf.

Liu, M., \& Peng, W. (2009). Cognitive and psychological predictors of the negative outcomes associated with playing MMOGs (massively multiplayer online games). Computers in Human Behavior, 25(1):1306-1311.

McClellan, J. E. III \& Dorn, H. (2008). Science and technology in world history. JHU Press.

Mehroof, M., \& Griffiths, M. D. (2010). Online gaming addiction: The role of sensation seeking, self-control, neuroticism, aggression, state anxiety, and trait anxiety. Cyberpsychology, Behavior, and Social Networking, 13(3):313-316. doi: 10. 1089/cyber.2009.0229

Meerkerk, G. J., Van den Eijnden, R. J. J. M., \& Garretsen, H. F. L. (2006). Predicting compulsive Internet use: It's all about sex! CyberPsychology \& Behavior, 9(1):95-103. doi: 10.1089/cpb.2006.9.95

$\mathrm{Ng} \mathrm{BD}$, Wiemer-Hastings P. Addiction to the internet and online gaming. Cyberpsychol Behav 2005; 8:110-113.10 
Niemz. K., Griffiths, M., \& Banyard, P. (2005). Prevalence of pathological Internet use among university students and correlations with self-esteem, the General Health Questionnaire (GHQ), and disinhibition. Cyberpsychol Behav. Dec, *.8, n.6, p. 56270 , 8(6):562-70. doi: $10.1089 / \mathrm{cpb} .2005 .8 .562$

Oliver, J. H. (2002). In Frans Mäyrä (Ed.), Proceedings of computer games and digital cultures conference. Tampere: Tampere University Press. Copyright: Authors and Tampere University Press.

Palfrey, J. \& Gasser, Urs. (2011). Nascidos na era digital: entendendo a primeira geração de nativos digitais. Porto Alegre: Artmed.

Peters, C.S., \& Malesky, L.A. (2008). Problematic usage among highly-engaged players of massively multiplayer online role playing games. Cyberpsychology \& Behavior, 11, 480-483. (1):480-483.

Park, J. H., Han, D. H., Kim, B. N., Cheong, J. H., \& Lee, Y. S. (2016). Correlations among social anxiety, self-esteem, impulsivity, and game genre in patients with problematic online game playing. Psychiatry Investigation, 13(3):297-304. doi:10.4306/pi.2016.13.3.297

Peukert, P., Sieslack, S., Barth, G., \& Batra, A. (2010) Internet and computer game addiction: phenomenology, comorbidity, etiology, diagnostics and therapeutic implications for the addictives and their relatives. Psychiatrische Praxis, 37(5):219-224. DOI: 10.1055/s-00301248442

Retirado de https://www.thewrap.com/what-is-a-video-game-a-short-explainer/

Peters, C.S., \& Malesky, L.A. (2008). Problematic usage among highly-engaged players of massively multiplayer online role playing games. Cyberpsychology \& Behavior, 11(1):480483.

Rice, R. A. (2006). MMO Evolution. Raleigh, NC: Lulu Press.

Sarsar, N. M. (2008). What children can learn from MMORPGs. ERIC, online submission, Available from: https://files.eric.ed.gov/fulltext/ED501741.pdf 
Smyth, J. (2007) Beyond self-selection in video game play: an experimental examination of the consequences of massively multiplayer online role-playing game play. Cyberpsychol Behav, 10(1):717-721.

Shaw, M., \& Black, D. W. (2008). Internet Addiction: Definition, Assessment, Epidemiology and Clinical Management. CNS Drugs, 22(1):353-365. http://dx.doi.org/10.2165/00023210$\underline{200822050-00001}$

Spada, M. M., \& Caselli, G. (2017). The metacognitions about online gaming scale: Development and psychometric properties. Addictive Behaviors, v.64, p.281 286. 64(1):2816. doi:10.1016/ j.addbeh.2015.07.007

Spritzer DT, Picon FA. Dependência de jogos eletrônicos. Porto Alegre: Artmed; 2012. p. 116-24.

Spritzer, D. T. \& Picon, F. (2013). Dependência de jogos eletrônicos. In: Vivendo esse mundo digital.p. 116-124. São Paulo: Artmed.

Stavropoulos, V., Kuss, D., Griffiths, M.D., \& Motti-Stefanidi, F. (2015a). A longitudinal study of adolescent Internet addiction: The role of conscientiousness and class-room hostility. Journal of Adolescent Researchhttp://dx.doi.org/10.1177/743558415580163.

Souza, B. C., Silva, L. X. D. L., \& Roazzi, A. (2010). MMORPGS and cognitive performance: A study with 1280 Brazilian high school students. Computers in Human Behavior, 26(6):1564-1573.

Stavropoulos, V., Kuss, D.J., Griffiths, M.D., Wilson, P.,\& Motti

Stavropoulos, V., Kuss, D., Griffiths, M.D., \& Motti-Stefanidi, F. (2015a). A longitudinal study of adolescent Internet addiction: The role of conscientiousness and class-room hostility. Journal of Adolescent Researchhttp://dx.doi.org/10.1177/743558415580163. 
Stefanidi, F. (2017). Mmorpg Gaming And Hostility Predict Internet Addiction Symptoms In Adolescents: An Empirical Multilevel Longitudinal Study. Addictive Behaviours, 64(1): 294-300, Doi:10.1016/J.Addbeh.2015.09.001

Tao, R., Huang, X., Wang, J., Zhang, H., Zhang, Y., \& Li, M. (2010). Proposed diagnostic criteria for internet addiction. Addiction, 105(3):556-564.

Tao R, Huang X, Wang J, Zhang H, Zhang Y, Li M. Proposed diagnostic criteria for internet addiction. Addiction. 2010 Mar; 105(3):556-64.

Taylor, T. L. (2006). Play Between Worlds: Exploring Online Game Culture. The MIT Press. Chicago.

Turkle, S. (1997) A vida no ecrã: a identidade na era da internet. Lisboa: Relógio D’Água.

Trepte, S., Reinecke, L., \& Juechems, K. (2012). The social side of gaming: How playing online computer games creates online and offline social support. Computers in Human Behavior, 28(3):832-839. doi: http://dx.doi.org/10.1016/j.chb.2011.12.003

Xiuqin, H., Huimin, Z., Mengchen, L., Jinan, W., Ying, Z., \& Ran, T. (2010). Mental health, personality, and parental rearing styles of adolescents with internet addiction disorder. Cyberpsychology Behavior and Social Networking, 13(1):401-406.

Wan, C., \& Chiou, W. (2006). Psychological Motives and Online Games Addiction: A Test of Flow Theory and Humanistic Needs Theory for Taiwanese Adolescents. CyberPsychology and Behavior, 9(1):317-324.

Van Eck, Richard. (2010). " Gaming and Cognition: Theories and Practice from the Learning Sciences." IGI Global. Cap. 3. Anderson, Bodi. "MMORPGs in support of Learning: Current Trends and Future Uses." 55-81 
Res., Soc. Dev. 2019; 8(10):e498101421

ISSN 2525-3409 | DOI: http://dx.doi.org/10.33448/rsd-v8i10.1421

Wei, H., Chen, M., Huang, P., \& Bai, Y. (2012). The association between online gaming, social phobia and depression: An internet survey. BMC Psychiatry, 12(1):929. $10.1186 / 1471-244 \mathrm{X}-12-92$

Whang, L. S. (2003). Online game dynamics in Korean society: Experiences and lifestyles in the online game world. Korea Journal, p.7-34.

Williams, D. et al. (2006). From tree house to barracks: the social life of guilds in world of warcraft. Games and Cultures, 1(4):338-361.

Wing, J. S. L. (2007). Interpersonal Relationships: From Aggression and Hatred to Altruism and Love - how should one perceive another in a computer game. Available em http://www.cs.mcgill.ca/ jlionw/ COMP763/report.pdf

Yen, J.Y., Ko, C.H., Yen, C.F., Wu, H.Y., \& Yang, M.J. (2007). The comorbid psychiatric symptoms of Internet addiction: attention deficit and hyperactivity disorder (ADHD), depression, social phobia, and hostility. Journal of Adolescent Health, 41(1):93-98.

Yen, J.Y., Liu, T. L., Wang, P.W., Chen, C.S., Yen, C.F., \& Ko, C.H. (2017). Association between internet gaming disorder and adult attention deficit and hyperactivity disorder and their correlates: Impulsivity and hostility. Addictive Behaviors, 64(1):308-313. doi: $\underline{10.1016 / j . a d d b e h .2016 .04 .024}$

Young, K. S. (2009). Internet addiction: diagnosis and treatament considerations. J. Contemp. Psychother, v. 39, p.241-246.

Zhang, F., \& Kaufman, D. (2016). Physical and cognitive impacts of digital games on older adults: A meta-analytic review. Journal of Applied Gerontology, 35(11):1189-1210. doi:10.1177/0733464814566678.

Zhong Z. J. (2011). The effects of collective MMORPG (Massively multiplayer online role playing games) play on gamers' online and offline social capital. Computers in Human Behavior, 27(1):2352-2363. doi: http://dx.doi.org/ 10.1016/j.chb.2011.07.014 


\section{Res., Soc. Dev. 2019; 8(10):e498101421}

ISSN 2525-3409 | DOI: http://dx.doi.org/10.33448/rsd-v8i10.1421

Zhong, Z., \& Yao, M. Z. (2012). Gaming motivations, avatar-self identification and symptoms of online game addiction. Asian Journal of Communication, http://dx.doi.org/10. $1080 / 01292986.2012 .748814$

\section{Porcentagem de contribuição de cada autor no manuscrito}

Paula Argemi Cassel-40\%

Thomaz Girardi Terribile $-40 \%$

Jéssica Costa Machado- 20\% 